\title{
GEOPHYSICAL EXCITATION OF NUTATION - COMPARISON OF DIFFERENT MODELS
}

\author{
Jan VONDRÁK* and Cyril RON
}

Department of Galaxies and Planetary Systems, Astronomical Institute, Academy of Sciences of the Czech Republic, Bočni II, 14100 Prague 4, Czech Republic, phone +420226258451

*Corresponding author's e-mail: vondrak@ig.cas.cz

\begin{tabular}{l} 
ARTICLE INFO \\
\hline Article history: \\
Received 3 January 2014 \\
Accepted 15 April 2014 \\
Available online 7 May 2014 \\
\hline Keywords: \\
Rotation of the Earth \\
Geophysical excitations \\
Geomagnetic jerks \\
Celestial pole offsets \\
Free core nutation
\end{tabular}

\begin{abstract}
We use the most recent models of excitations by geophysical fluids (atmosphere, oceans) to derive their impact on the motion of the Earth spin axis in space - nutation. Celestial pole offsets (i.e., the differences between the observed nutation and the adopted IAU model) due to these geophysical excitations are computed for a non-rigid Earth model to account for the realistic Earth's response. The results, corresponding to excitations provided by different agencies, are compared with the celestial pole offsets observed by Very Long-Baseline Interferometry. It is demonstrated that the celestial pole offsets excited by different models of geophysical fluids differ. Amplitudes based on European models ERA are larger than the observed ones, U.S. model NCEP/NCAR yields better agreement. If we apply re-initialization of the integration at epochs of geomagnetic jerks, the agreement between the integrated and observed values is much improved for all models studied.
\end{abstract}

\section{INTRODUCTION}

The effects of geophysical excitations in nutation are caused by quasi-diurnal changes of angular momentum functions of the atmosphere and oceans, expressed in terrestrial frame. Therefore, highresolution data are needed, with sub-diurnal sampling. Fortunately, the atmospheric/oceanic data with 6-hour steps are now available, from different agencies, which enables these studies to be made.

In our recent study (Vondrák and Ron, 2010) we demonstrated that the atmospheric and oceanic excitations, playing a dominant role in polar motion and rotational velocity of the Earth, have also a nonnegligible and observable effect in nutation, i.e., the quasi-periodic motion of Earth's axis of rotation in space, especially at annual and semi-annual frequencies. However, this effect was found to be different for different sources of atmospheric/oceanic angular momentum functions (Ron et al., 2011). Better agreement with Very Long-base Interferometry (VLBI)-based celestial pole offsets (CPO) was achieved for the atmospheric/oceanic data from U.S. agency (NCEP + ECCO) than from the European one (ERA + OMCT). We also studied the coherence between geophysically excited and observed nutation (Ron and Vondrák, 2011); the results confirmed our previous findings. Very recently Malkin (2013) implied that the observed sudden changes of Free Core Nutation (FCN) amplitude and phase were probably related to the epochs of geomagnetic jerks. Therefore we tested this possibility by re-initializing numerical integration at these epochs to see if the agreement between the observed and excited CPO is improved. Preliminary and promising results are presented by Ron et al. (2014). The present paper continues in these efforts, namely by comparing the geophysically excited CPO, based on the most recent data from different sources, with their values observed by VLBI, and also by studying possible impacts by geomagnetic jerks.

\section{THE INPUT DATA}

We use two different kinds of input data (celestial pole offsets and geophysical excitations) in our study, all covering the period 1989.0-2013.5:

- Celestial pole offsets, taken from the most recent IVS combined solution (Schlüter and Behrend, 2007) ivs $13 q 2 x$. Original data are given at unequal intervals (typically 1-7 days long), sometimes with large deviations. There exist many solutions provided by different VLBI analysis centers, but, as demonstrated by Malkin (2012), the IVS series may be preferable for some applications, as e.g. FCN models.

- Geophysical excitations were used from three different models, provided by two sources, available at Data Center of the IERS; all are given in strictly 6-hour intervals:

$\circ$ Pressure (matter) and wind (motion) terms of atmospheric angular momentum functions (AAMF) from NCEP/NCAR re-analysis (Salstein, 2005). Instead of oceanic excitations from ECCO model (Gross et al., 2005) that we found to have very low diurnal and sub-diurnal signal (Vondrák and Ron, 2010), we use here the atmospheric pressure terms with inverted barometer corrections (Wunsch and Stammer, 1997). This model represents a very simple solution of oceanic response to the changes of 


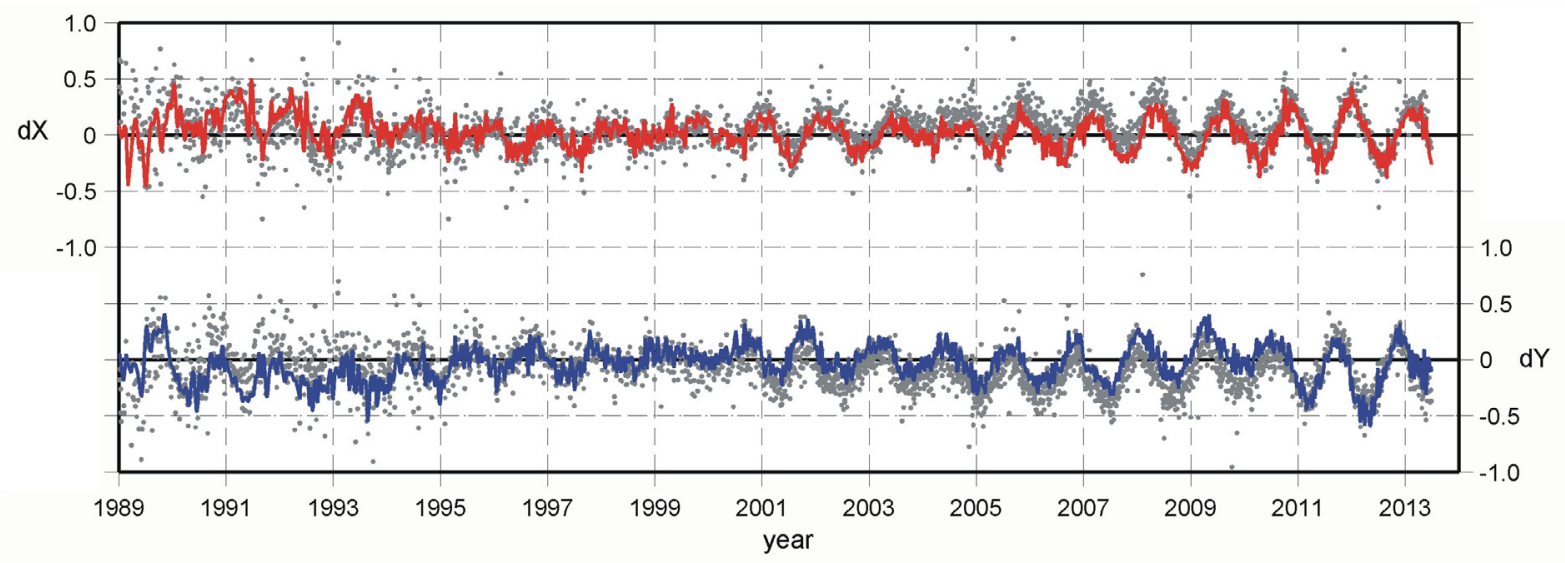

Fig. 1 IVS celestial pole offsets in mas (dots) and their filtered values (full lines).

atmospheric pressure variations over the oceans.

$\circ$ Pressure and wind terms of AAMF plus matter and motion terms of oceanic angular momentum functions (OAMF) from the European Centre for Medium Weather Forecast (ECMWF) ERAinterim analysis model for the atmosphere and oceanic model OMCT driven by the same atmospheric model, prepared at IERS/GFZ center (Thomas et al., 2006; Dobslaw et al., 2010).

$\circ$ Pressure and wind terms of AAMF plus matter and motion terms of OAMF from ECMWF reanalysis ERA40 model, completed by operational analysis after 2001.0; oceanic model OMCT is driven by the same atmospheric model, prepared at IERS/GFZ center.

Celestial pole offsets (CPO) are the differences between the observed pole position in space and its adopted model (IAU 2000 for nutation and IAU 2006 for precession). Thus they contain Free Core Nutation (FCN), deficiencies of the model used, and also so called Sun-synchronous (prograde annual) term, part of the IAU 2000 model which is supposed to account for the atmospheric effects. CPO data were cleaned (by removing all values larger than 1mas), and then filtered (using the smoothing by Vondrák (1977)) to contain only the periods between 60 and 6000 days. The periods shorter than 60 days were removed to suppress the short-periodic noise of the observations, and periods longer than 6000 days were removed because studying long-periodic variations is beyond the scope of this paper. The original IVS observations and filtered data are displayed in Figure 1; all values are given in milliarcseconds (mas).

Geophysical angular momentum functions are given in the rotating terrestrial frame. Only the first two components $\chi_{1}, \chi_{2}$ (around equatorial axes), are important for exciting nutation, the third one, $\chi_{3}$ (around polar axis), influences only the speed of rotation. Only the quasi-diurnal retrograde motion (that becomes long-periodic in celestial frame) is capable of exciting nutation. Here we use the complex values $\chi=\chi_{1}+i \chi_{2}$ that were subject to demodulation
(Brzeziński et al., 2002) at the retrograde diurnal frequency. We first removed a constant part (leading to a diurnal signal after demodulation) and then used a simple formula $\chi^{\prime}=-\chi e^{i \phi}$, where $\varphi$ is the Greenwich sidereal time, to transform the excitations into the celestial reference frame. Because we are interested only in long-periodic motion near nutation frequencies, we also applied the smoothing (Vondrák, 1977) to remove all periods shorter than 10 days. The three time series of excitations, mentioned above (i.e., NCEP, ERA and ERA40), after demodulation are graphically displayed in Figures $2-4$ (atmospheric and oceanic terms being added in the latter two models). Dimensionless values $\chi_{1}, \chi_{2}$, are given in units of $10^{-8}$.

In all three series wind/motion terms, which are much larger than the pressure/matter terms, are similar. However, the pressure/matter terms have slightly larger amplitudes in both European models than in the NCEP/NCAR data.

\section{COMPARISONS}

We compare the series of geophysical excitations with celestial pole offsets, using two different methods:

- Spectral analysis by FFT is used, and the amplitude spectrum of the IVS CPO is compared with the spectra of geophysical excitations, multiplied by Brzeziński transfer function (Brzeziński, 1994) for celestial frequency $\sigma$ :

$$
T_{p, w}(\sigma)=\sigma_{C}\left(\frac{1}{\sigma_{C}^{\prime}-\sigma}+\frac{a_{p, w}}{\sigma_{f}^{\prime}-\sigma}\right) \text {, }
$$

where

$\sigma_{C}^{\prime}=6.32000+0.00237 i, \sigma_{f}^{\prime}=-0.0146011+0.0001533 i$

(in radians per sidereal day) are the complex Chandler and FCN frequencies in celestial frame, respectively, $\sigma_{C}=0.01962+0.00237 i$ is the complex Chandler frequency in terrestrial frame and $a_{p}=9.509 \times 10^{-2}, a_{w}=5.489 \times 10^{-4}$ are dimensionless constants. The transfer function is 


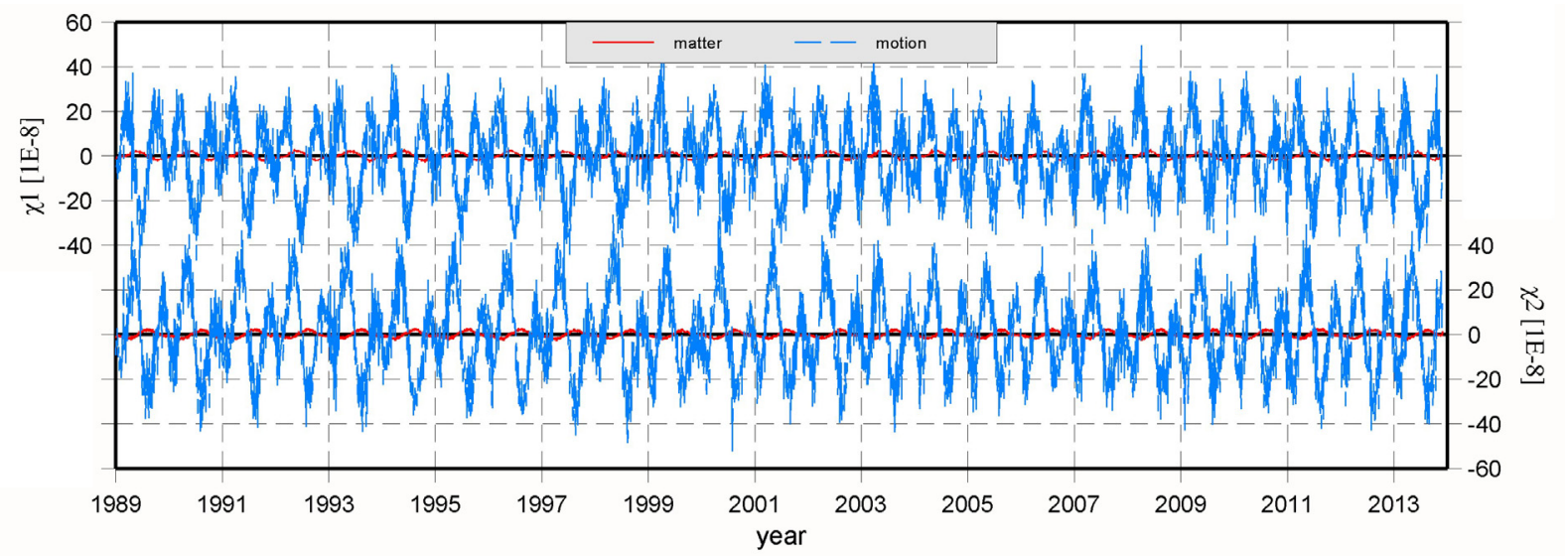

Fig. 2 Atmospheric angular momentum functions from NCEP/NCAR model.

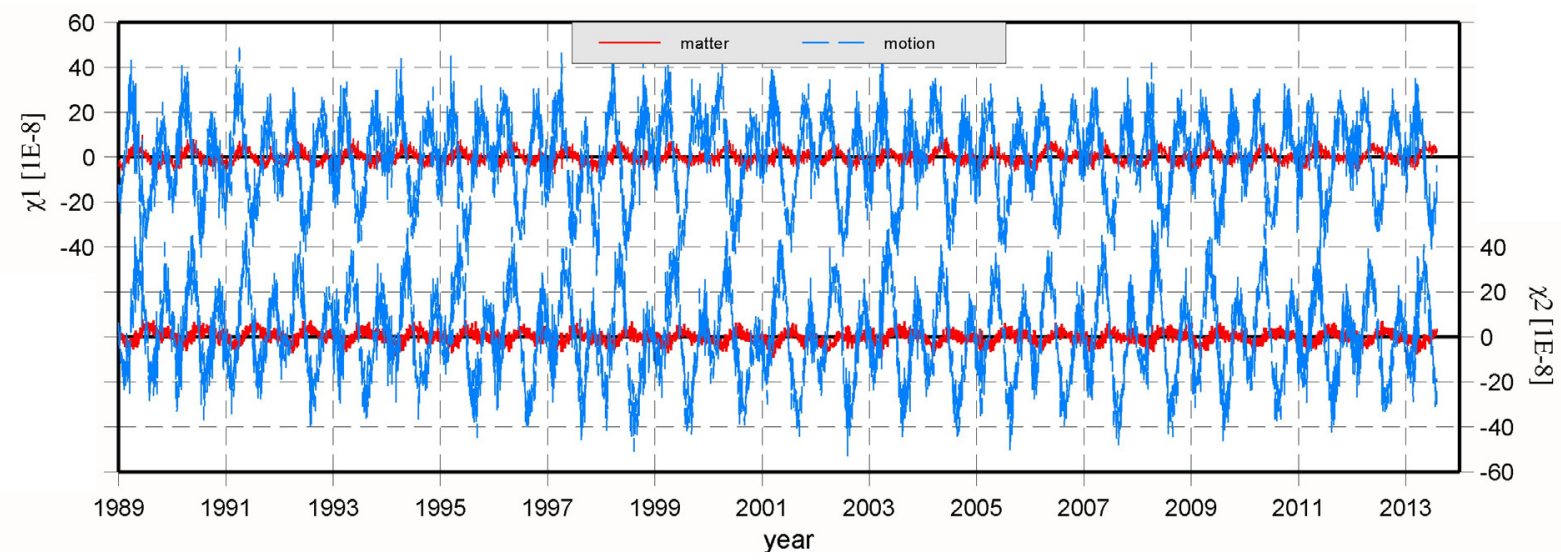

Fig. 3 Atmospheric + oceanic angular momentum functions from ERA-interim model.

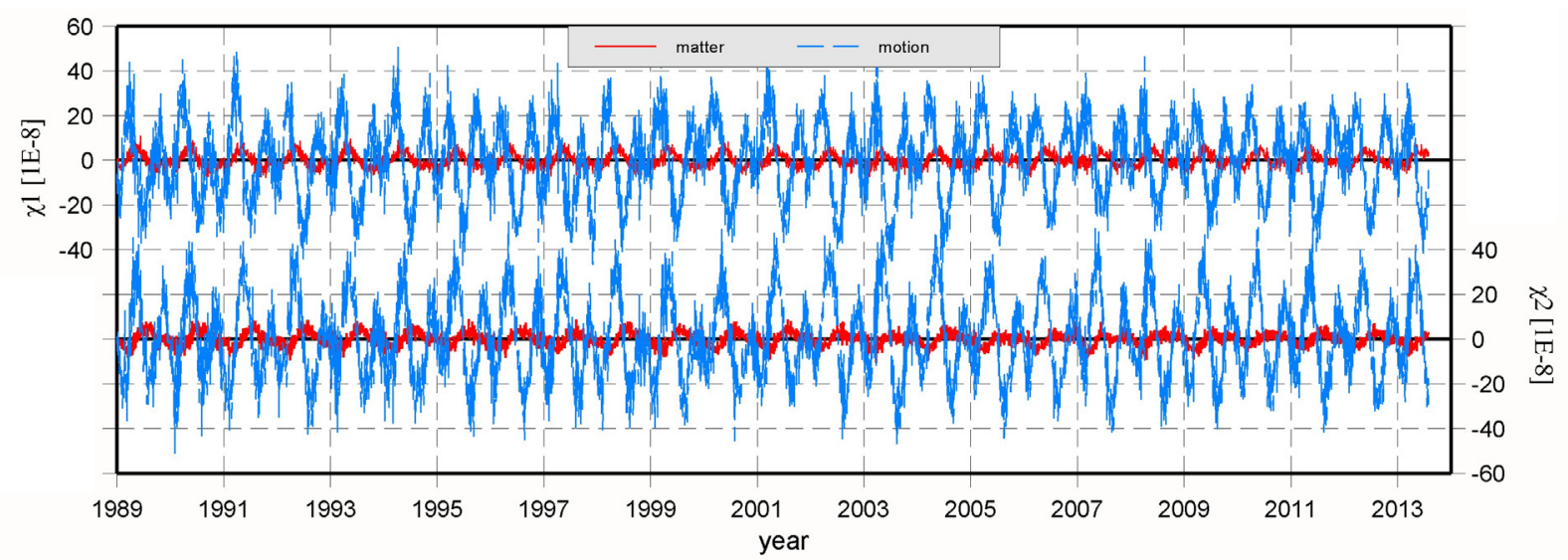

Fig. 4 Atmospheric + oceanic angular momentum functions from ERA40 model.

evidently different for pressure/matter $(p)$ and wind/motion $(w)$ term.

- Numerical integration of the Brzeziński (1994) broad-band Liouville second-order differential equations in complex form, expressed in celestial frame:

$$
\begin{aligned}
& \ddot{P}-i\left(\sigma_{C}^{\prime}+\sigma_{f}^{\prime}\right) \dot{P}-\sigma_{C}^{\prime} \sigma_{f} P= \\
& \quad=-\sigma_{C}\left\{\begin{array}{c}
\sigma_{f}^{\prime}\left(\chi_{p}^{\prime}+\chi_{w}^{\prime}\right)+\sigma_{C}^{\prime}\left(a_{p} \chi_{p}^{\prime}+a_{w} \chi_{w}^{\prime}\right)+ \\
+i\left[\left(1+a_{p}\right) \dot{\chi}_{p}^{\prime}+\left(1+a_{w}\right) \dot{\chi}_{w}^{\prime}{ }_{w}\right]
\end{array}\right\},
\end{aligned}
$$

in which $P=d X+i d Y$ is the excited motion of Earth's spin axis in celestial frame. To integrate 


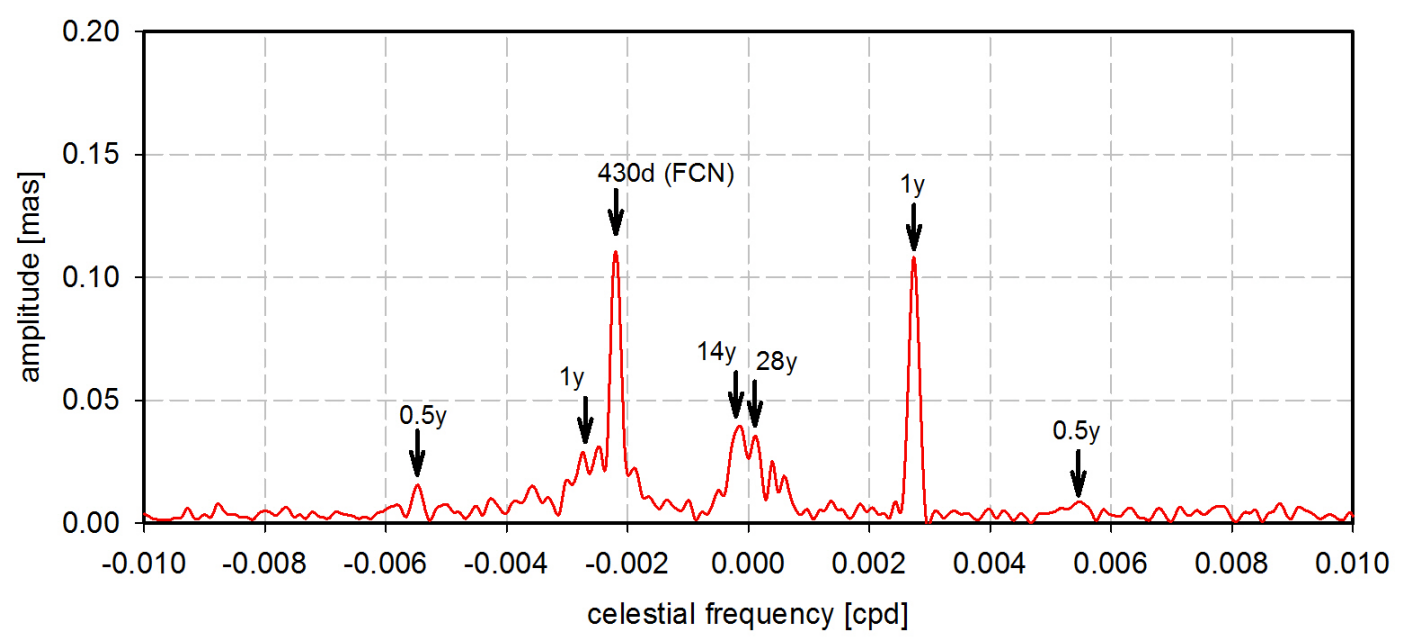

Fig. 5 Amplitude spectrum of IVS celestial pole offsets.

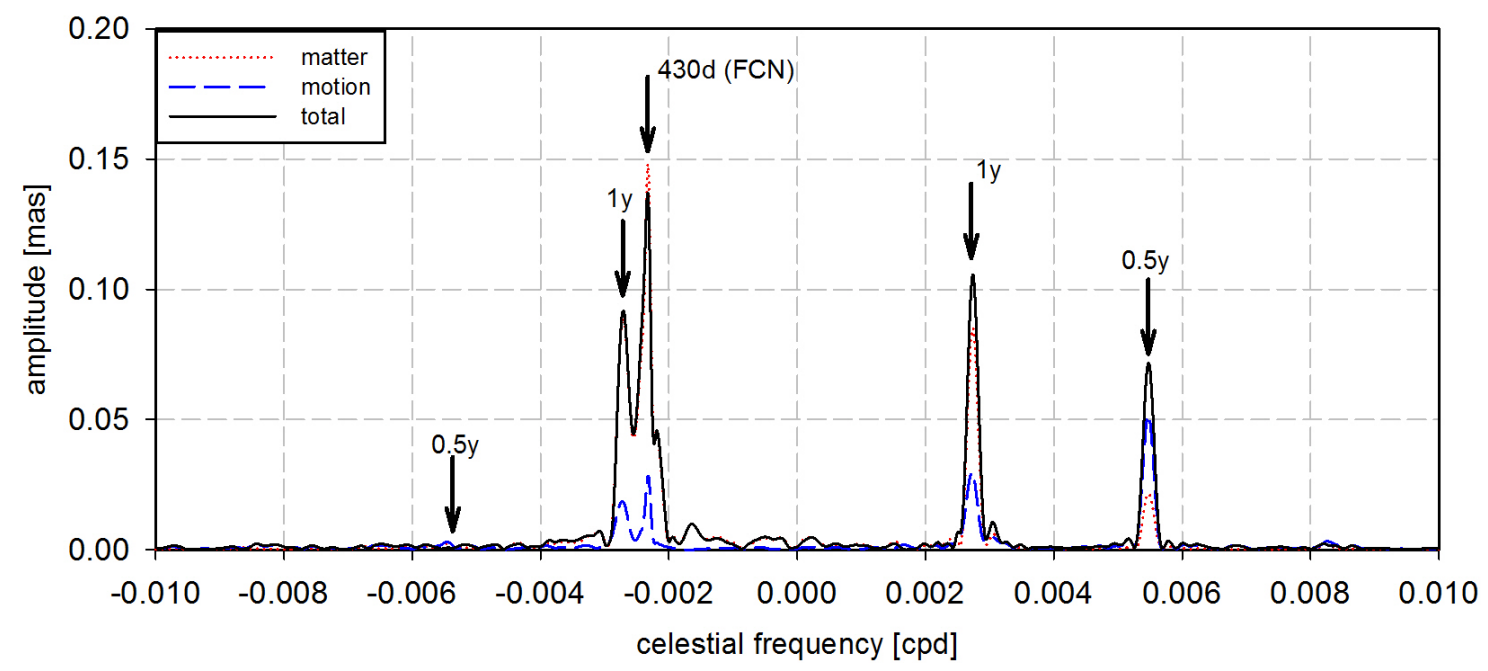

Fig. 6 Amplitude spectrum of NCEP/NCAR pressure term with IB correction, multiplied by Brzeziński transfer function.

the system, we use the subroutine $r \mathrm{k} 4$ for fourthorder Runge-Kutta method (Press et al., 1992) with 6-hour steps, which we modified into complex form. Before doing the integration, we need to choose the initial complex values of pole position and its first derivative $P_{0}, \dot{P}_{0}$. They represent four integration constants that are closely tied with the phases and amplitudes of the two free modes of the solution (Chandler wobble and Free Core Nutation). We constrain the latter one,

$\dot{P}_{0}=i \sigma_{f}^{\prime} P_{0}$

so that the unnecessary free Chandlerian amplitude (that has prograde nearly-diurnal period in celestial frame) disappears. It is necessary to say that the choice of $P_{0}$, with the constraint above, affects only the free part of the solution, the forced one remains the same.

\subsection{COMPARISON OF THE SPECTRA}

The spectra of IVS celestial pole offsets and all three geophysical excitations, multiplied by Brzeziński transfer function, are displayed in Figures $5-8$.

Figure 5 shows the amplitude spectrum of IVS CPO. IAU 2000 model of nutation contains also a small so called Sun-synchronous (prograde annual) empirical correction that is supposed to account for the atmospheric effects (Mathews et al., 2002). Consequently, we added the correction $(0.1082+0.0104 i) e^{i l^{\prime}}$ (in mas), where $l$ ' stands for the mean anomaly of the Sun, to the complex values of CPO values (real part to $\mathrm{d} X$, imaginary part to $\mathrm{d} Y$ ), so that they are directly comparable to the geophysical effects. The figure depicts the dominant peak at retrograde FCN frequency (period of about 430 days); similar peak exists at prograde annual frequency, smaller peaks are present also at retrograde annual and 


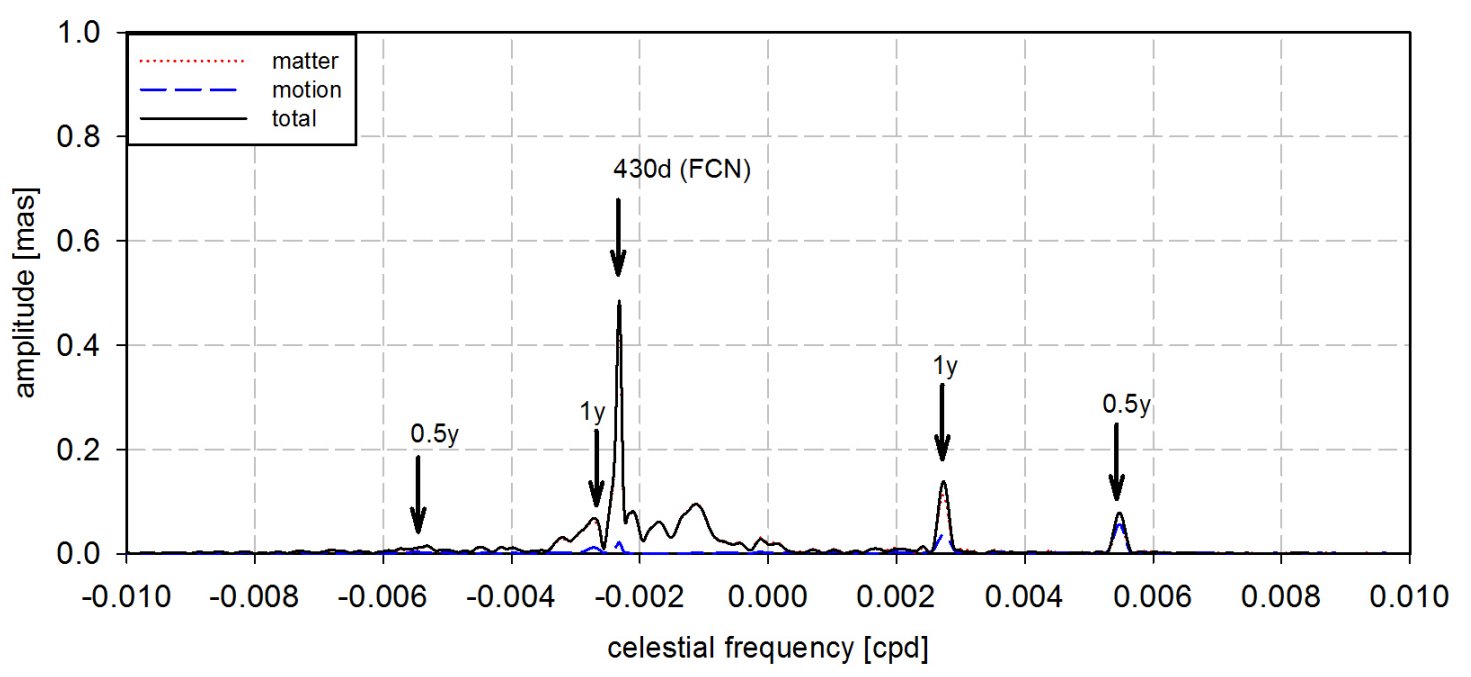

Fig. 7 Amplitude spectrum of ERA-interim atmospheric + oceanic excitations, multiplied by Brzeziński transfer function.

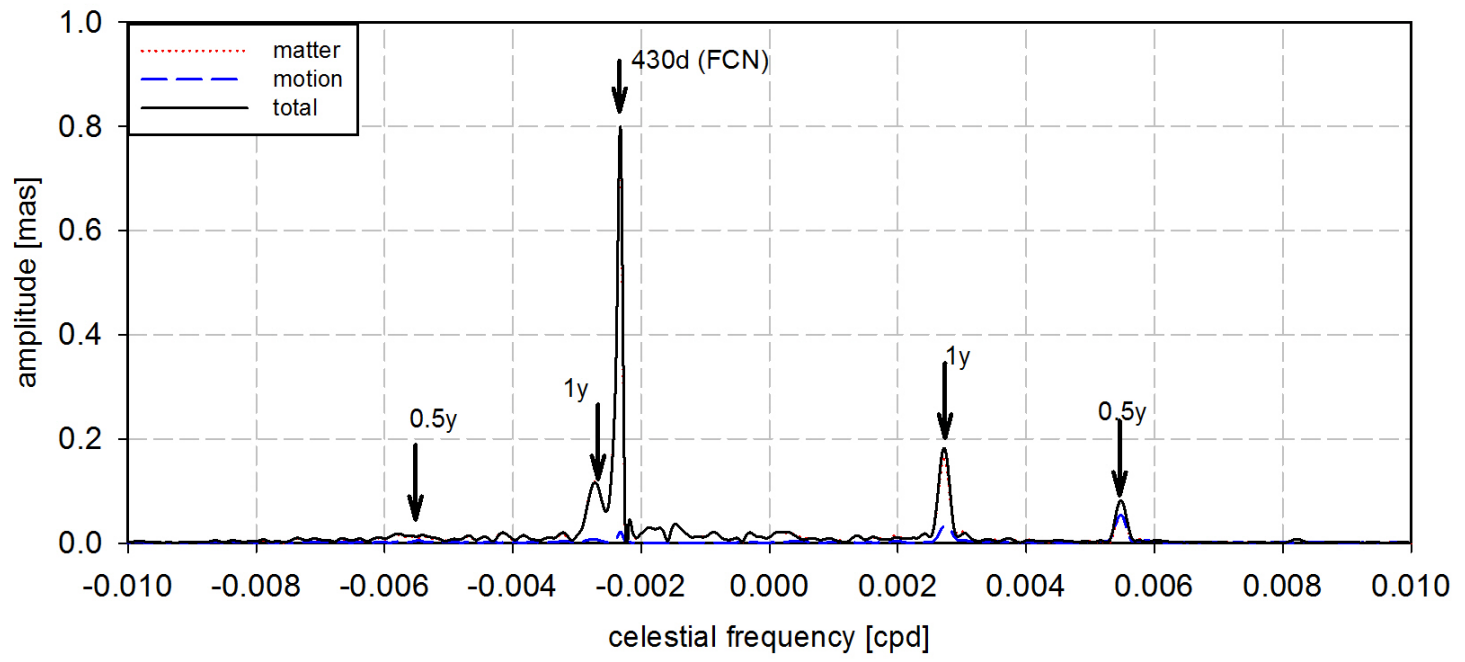

Fig. 8 Amplitude spectrum of ERA40 atmospheric + oceanic excitations, multiplied by Brzeziński transfer function.

semi-annual frequencies, and at long-periodic part of the spectrum. The spectra of geophysical excitation functions, multiplied by Brzeziński transfer function, are shown in Figures 6, 7 and 8 for above mentioned NCEP, ERA-interim and ERA40 solutions, respectively.

The spectrum of NCEP solution is comparable to the one of the observed CPO, but both ERA solutions, especially ERA40 in Figure 8, yield much higher amplitudes (notice the difference in scale of Figures 5 and 6 on one side, and Figures 7 and 8 on the other one).

\subsection{COMPARISON OF THE INTEGRATED VALUES WITH OBSERVATIONS}

Geophysically excited motion of celestial pole, obtained by numerical integration of $\mathrm{Eq}$ (2), is compared with the IVS solution of CPO, corrected for Sun-synchronous correction (see above). To obtain the best fit to so corrected $\mathrm{CPO}$ values, the integration was repeated with different initial values $P_{0}$. To find the minimum rms fit, many complex values in a net surrounding the observed value were tried; the time derivative at the initial epoch was calculated using $\mathrm{Eq}(3)$.

The results obtained with NCEP, ERA-interim and ERA40 excitations are graphically depicted in Figures 9 - 11, respectively. Evidently, the NCEP excitations yield the best agreement with observations, while both European models give systematically larger amplitudes. In all three cases phase differences between integration and observation are present, leading to rather high $\mathrm{rms}$ fit and low correlation between the integrated and observed series.

\subsection{GEOMAGNETIC JERKS}

Geomagnetic jerks (GMJ) are rapid changes of the secular variations of geomagnetic field. They were 


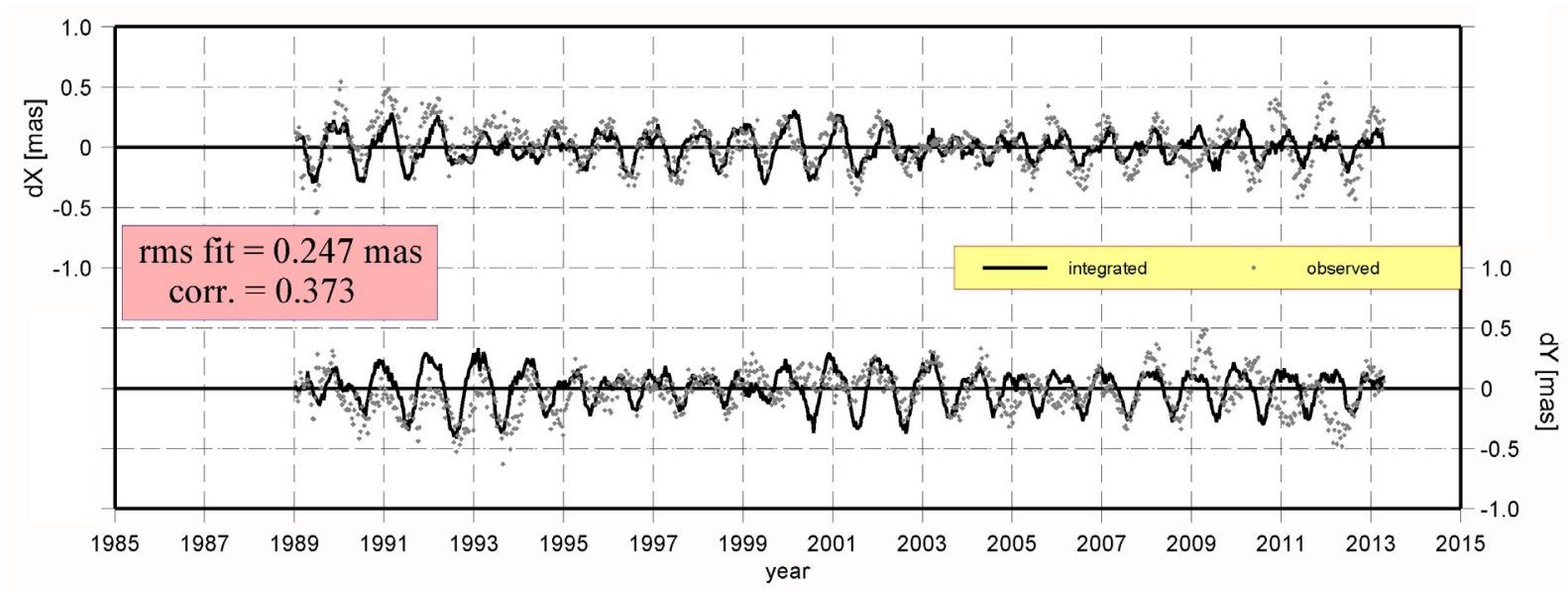

Fig. 9 Observed and integrated celestial pole offsets obtained with NCEP/NCAR data.

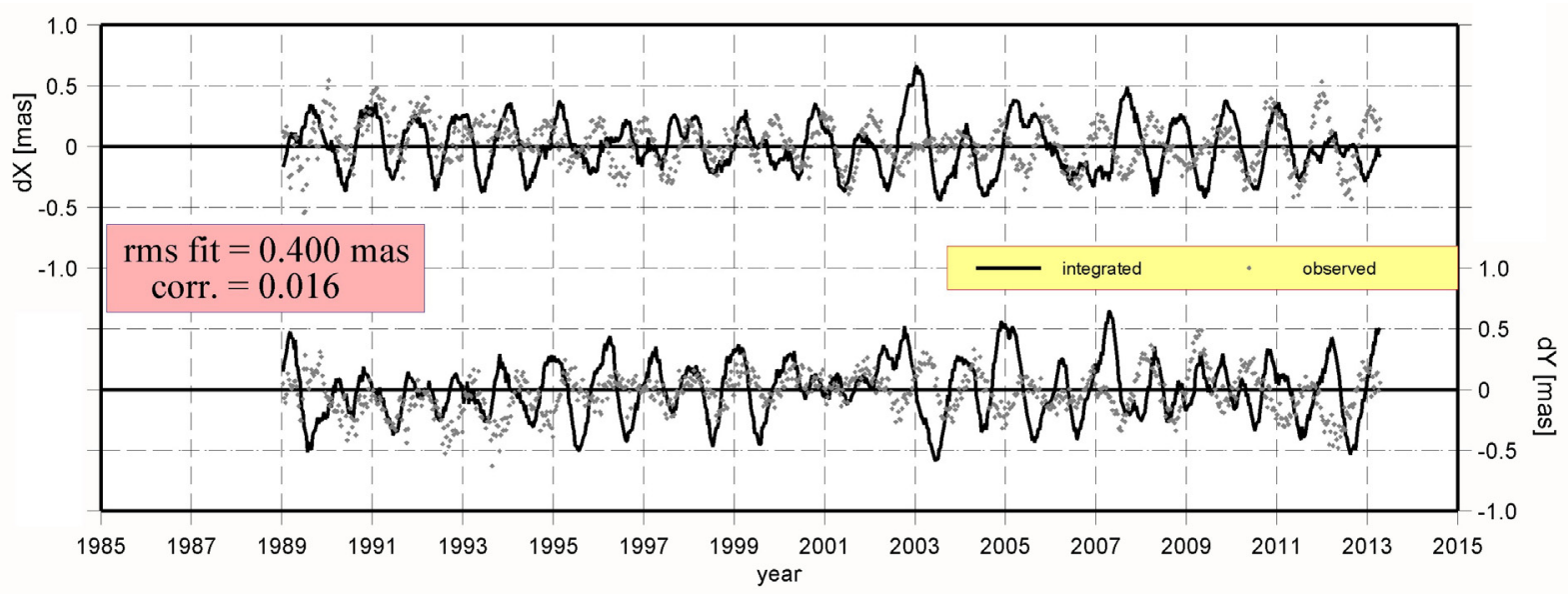

Fig. 10 Observed and integrated celestial pole offsets obtained with ERA-interim data.

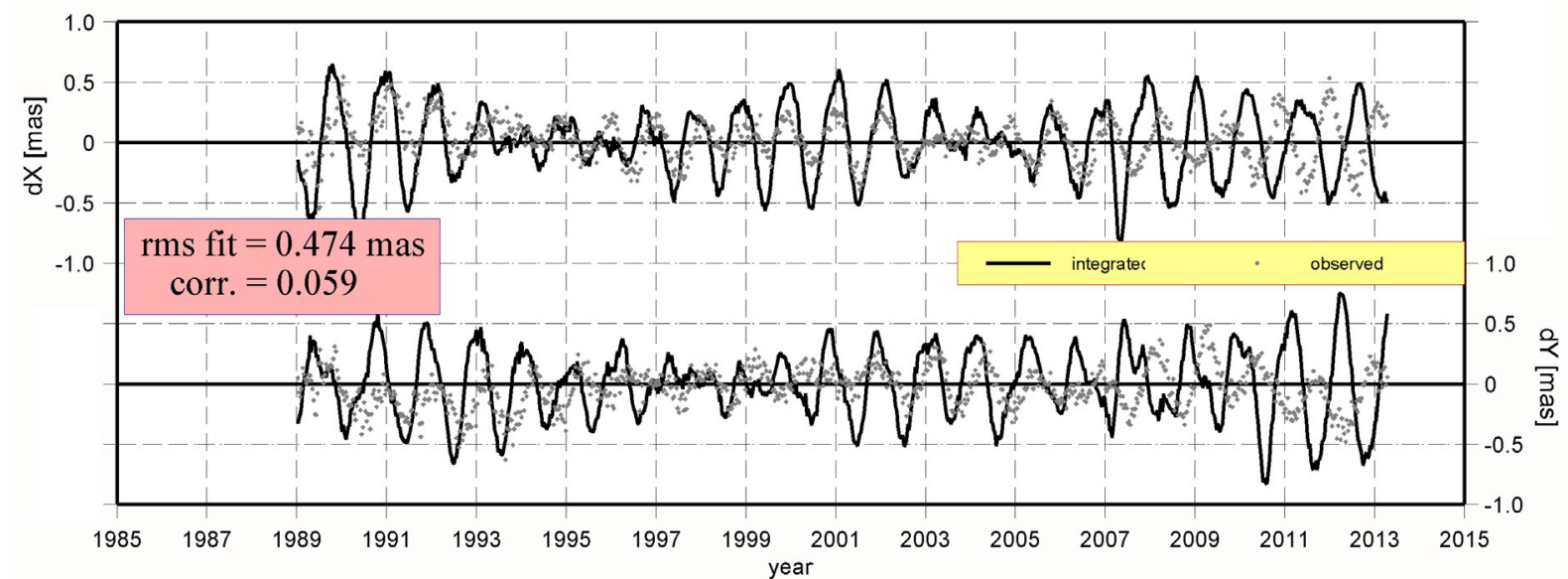

Fig. 11 Observed and integrated celestial pole offsets obtained with ERA40 data.

recently indicated by Malkin (2013) as possible sources of changes of FCN parameters (amplitude, phase). The epochs of GMJ given by Malkin, who used the data published by Nagao et al. (2002), Olsen and Mandea (2007), Mandea et al. (2010), Chulliat et al. (2010), Kotzé et al. (2011), Silva and Hulot (2012), and Pavón-Carrasco et al. (2013), are as follows: 1994.0, 1999.0, 2003.5, and 2007.5. At each of these epochs we estimated the new initial position $P_{0}$, using the procedure described above in Section 3.2. By doing this, we introduce sudden steps in pole position at these epochs. We also tried to move slightly the epochs of re-initializing the integration around the GMJ epochs and found that the best fit (for NCEP excitation) is achieved for epochs shifted forward by 100 days; namely for 1994.27, 1999.27, 2003.77 and 


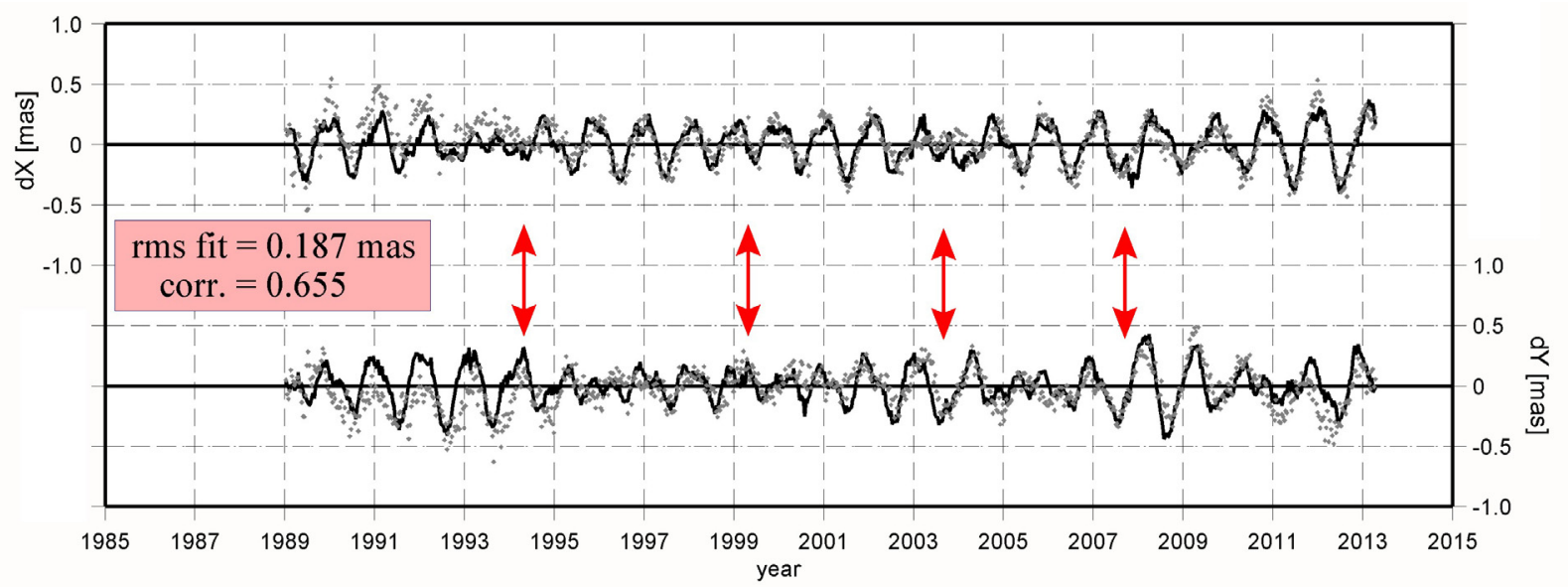

Fig. 12 Integrated CPO with NCEP data, new initial positions at GMJ epochs plus 100 days.

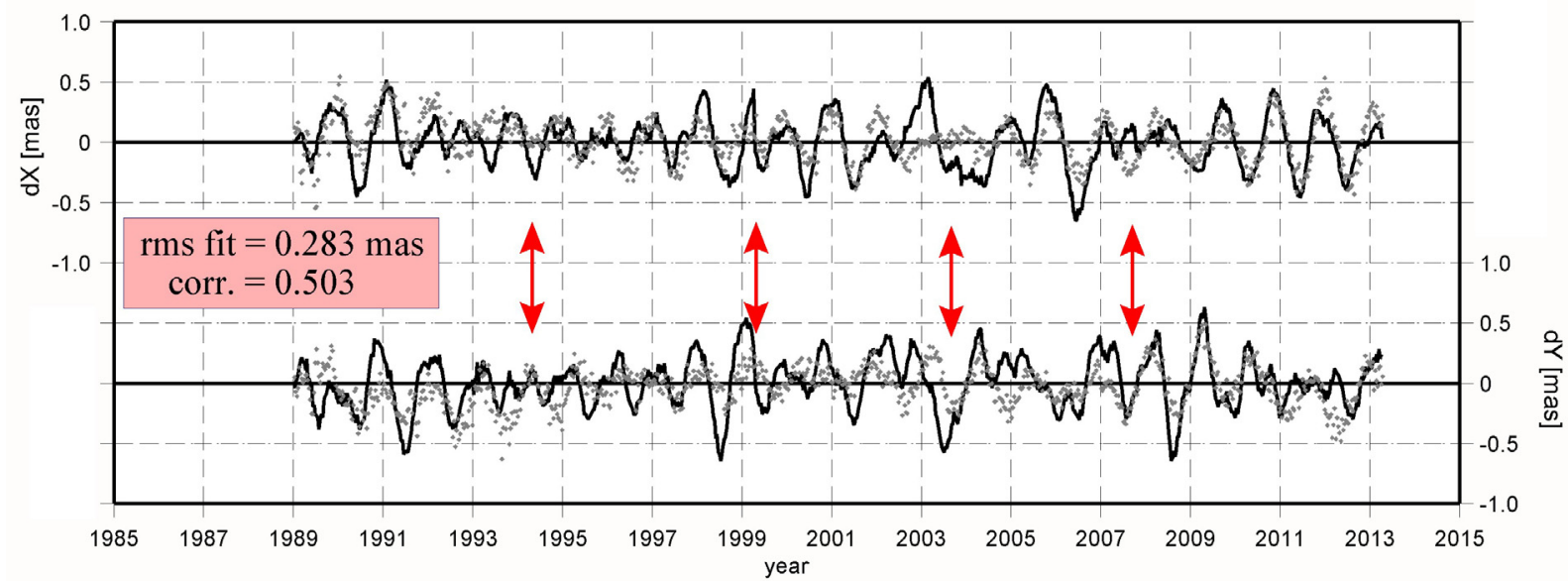

Fig. 13 Integrated CPO with ERA-interim data, new initial positions at GMJ epochs plus 100 days.

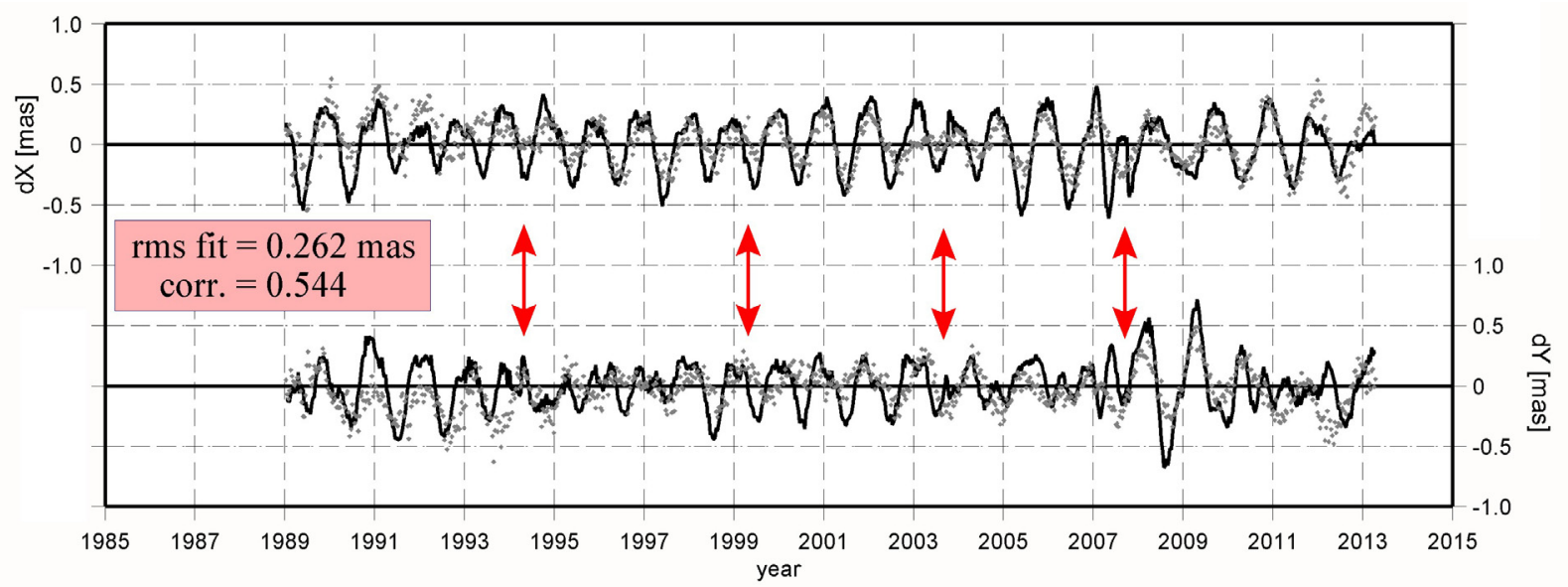

Fig. 14 Integrated CPO with ERA40 data, new initial positions at GMJ epochs plus 100 days.

2007.77. The results are demonstrated in Figures $12-$ 14 , in which the epochs of new initialization are marked by arrows, both rms fit and correlation between the two series are also displayed. This approach improves the fit to VLBI-based CPO essentially. NCEP excitations still keep the best fit to observations, but the improvement for both European models is also evident and substantial.

\section{CONCLUSIONS}

We demonstrate that the geophysical excitations yield significant and now observable contribution to nutation, of the order of 0.1 mas. Excitation functions display much higher power in motion/wind term than in matter/pressure one, but their influence on nutation is much smaller, due to a smaller multiplicative coefficient $a_{w}$ in Eq. (2). However, the nutations 
excited by the atmosphere + oceans differ substantially for different models of excitation studied here. When compared with VLBI-based celestial pole offsets, especially both European models yield systematically larger amplitudes. The application of re-initialization of numerical integration at the epochs 100 days after geomagnetic jerks improves the agreement with observations substantially. The best agreement is obtained with NCEP/NCAR model, in which the oceanic response to atmospheric pressure changes is modeled by a simple inverted barometer correction, and the effect of oceanic currents is neglected.

\section{ACKNOWLEDGEMENTS}

This research was financially supported by the grant No. 13-15943S "Geophysical excitations in the motion of Earth's axis of rotation", awarded by the Grant Agency of the Czech Republic. The help of both anonymous referees in improving the text is highly appreciated.

\section{REFERENCES}

Brzeziński, A.: 1994, Polar motion excitation by variations of the effective angular momentum function: II. Extended Model. Manuscripta Geodaetica, 19, 157171.

Brzeziński, A., Bizouard, C. and Petrov, S.: 2002, Influence of the atmosphere on Earth rotation: what new can be learned from the recent atmospheric angular momentum estimates? Surveys in Geophysics 23, No. 1, 33-69. DOI: 10.1023/A:1014847319391

Chulliat, A., Thébault, E. and Hulot, G.: 2010, Core field acceleration pulse as a common cause of the 2003 and 2007 geomagnetic jerks. Geophys. Res. Lett. 37, L07301. DOI: 10.1029/2009GL042019

Dobslaw, H., Dill, R., Grotzsch, A., Brzezinski, A. and Thomas, M.: 2010, Seasonal polar motion excitation from numerical models of atmosphere, ocean, and continental hydrosphere. J. Geophys. Res 115, B10406. DOI: 10.1029/2009B007127

Gross, R. S., Fukumori, I. and Menemenlis, D.: 2005, Atmospheric and oceanic excitation of decadal-scale Earth orientation variations. J. Geophys. Res. 110, B09405. DOI: 10.1029/2004JB003565

Kotzé, P. Corte, M. and Mandea, M.: 2011, Polynomial modelling of Southern African secular variation observations since 2005. Data Sci. J. 10, 95-101. DOI: 10.2481/dsj.IAGA-16

Malkin, Z.: 2012, Celestial pole offsets: From initial analysis to end user. Proc. IVS 2012 General Meeting, 375-379.

Malkin, Z.: 2013, Free core nutation and geomagnetic jerks. J. Geodyn. 72, 53-58. DOI: 10.1016/j.jog.2013.06.001

Mandea, M., Holme, R., Pais, A., Pinheiro, K., Jackson, A. and Verbanac, G.: 2010, Geomagnetic jerks: Rapid core field variations and core dynamics. Space Sci. Rev. 155, 147-175. DOI: 10.1007/s11214-010-9663-x

Mathews, P.M., Herring, T.A. and Buffet, B.A.: 2002, Modeling of nutation-precession for nonrigid Earth, and insights into the Earth's interior, J. Geophys. Res. 107, B4. DOI:10.1029/2001JB000390
Nagao, H., Higuchi, T., Iyemori, T. and Araki, T.: 2002, Automatic detection of geomagnetic jerks by applying a statistical time series model to geomagnetic monthly means. Progresses in Discovery Science. Lect. Notes Comp. Sci. 2281, 360-371.

Olsen, N. and Mandea, M.: 2007, Investigation of a secular variation impulse using satellite data: the 2003 geomagnetic jerk. Earth Planet. Sci. Lett. 255, 94-105. DOI: $10.1016 /$ j.epsl.2006.12.008

Pavón-Carrasco, F.J., Torta, J.M., Catalán, M., Talarn, A. and Ishihara, T.: 2013, Improving total field geomagnetic secular variation modeling from a new set of cross-over marine data. Phys. Earth Planet. Inter. 216, 1041-1052.

DOI: 10.1016/j.pepi.2013.01.002

Press, W.H., Teukolsky, S.A., Vetterling and W.T, Flannery, B.P.: 1992, Numerical Recipes in Fortran 77. The Art of Scientific Computing, $2^{\text {nd }}$ Edition, Cambridge University Press.

Ron, C. and Vondrák, J.: 2011, Coherence between geophysical excitations and celestial pole offsets. Acta Geodyn. Geomater. , No. 3, 243-247.

Ron, C., Vondrák, J. and Štefka, V.: 2011, Comparison of the various atmospheric and oceanic angular momentum series, in: N. Capitaine (ed.) Proc. Journées 2010 Systèmes de référence spatiotemporels, Observatoire de Paris, 221-222.

Ron, C., Vondrák, J. and Chapanov, Ya.: 2014, Free core nutation - possible causes of changes of its phase and amplitude, in: N. Capitaine (ed.) Proc. Journées 2013 Systèmes de référence spatio-temporels, Observatoire de Paris, in press.

Salstein, D.: 2005, Computing atmospheric excitation functions for Earth rotation/polar motion. Cahiers du Centre Européen de Géodynamique et de Séismologie 24, Luxembourg, 83-88.

Schlüter, W. and Behrend, D.: 2007, The International VLBI Service for Geodesy and Astrometry (IVS): Current capabilities and future prospects. J. Geod. 81, 379-387. DOI: 10.1007/s00190-006-0131-z

Silva, L. and Hulot, G.: 2012, Investigating the 2003 geomagnetic jerk by simultaneous inversion of the secular variation and acceleration for both the core flow and its acceleration, Phys. Earth Planet. Inter. 198, 28-50. DOI: 10.1016/j.pepi.2012.03.002

Thomas, M., Dobslaw, H. and Soffel, M.: 2006, The ocean's response to solar thermal and gravitational tides and impacts on EOP, In: A. Brzeziński, N. Capitaine, and B. Kołaczek (eds.), Proc. Journées 2005 Systèmes de référence spatio-temporels, Space Research Centre Warsaw and Observatoire de Paris, 203-206.

Vondrák, J.: 1977, Problem of smoothing of observational data II. Bull. Astron. Inst. Czechosl, 28, 84-89.

Vondrák, J. and Ron, C.: 2010, Study of atmospheric and oceanic excitations in the motion of Earth's spin axis in space. Acta Geodyn. Geomater. 7, No. 1, 19-28.

Wunsch, C. and Stammer, D.: 1997, Atmospheric loading and the oceanic "inverted barometer" effect. Reviews of Geophysics 35, 79-107. DOI: 10.1029/96RG03037 